\title{
Harmonic functions with missing coefficients
}

\author{
Jacek Dziok \\ Faculty of Mathematics and Natural Sciences, University of Rzeszów, ul. Prof. Pigonia 1, 35-310 \\ Rzeszów, Poland
}

\begin{abstract}
In the paper we introduce the classes of functions with missing coefficients defined by generalized Ruscheweyh derivatives and we show that they can be presented as dual sets. Moreover, by using extreme points theory, we obtain estimations of classical convex functionals on the defined classes of functions. Some applications of the main results are also considered.
\end{abstract}

Mathematics Subject Classification (2010). 30C55, 30C45

Keywords. harmonic functions, subordination, missing coefficients, Ruscheweyh operator, dual sets, correlated coefficients

\section{Introduction}

A complex-valued harmonic mapping $f$ in the open unit disk $\mathbb{U}:=\{z \in \mathbb{C}:|z|<1\}$ has a canonical decomposition

$$
f=h+\bar{g},
$$

where $h$ and $g$ are analytic functions in $\mathbb{U}$. We call $h$ the analytic part and $g$ the co-analytic part of $f$, respectively. Throughout this paper, we will discuss harmonic mappings that are sense-preserving in $\mathbb{U}$. By a theorem of Lewy [15], necessary and sufficient condition for $f$ to be locally univalent and sense-preserving in $\mathbb{U}$ is that

$$
\left|h^{\prime}(z)\right|>\left|g^{\prime}(z)\right| \quad(z \in \mathbb{U}) .
$$

Let $\mathcal{H}$ denote the class of sense-preserving harmonic functions in the unit disc $\mathbb{U}$. Any function $f \in \mathcal{H}$ can be written in the form

$$
f(z)=\sum_{n=0}^{\infty} a_{n} z^{n}+\sum_{n=1}^{\infty} \overline{b_{n} z^{n}} \quad(z \in \mathbb{U}) .
$$

Let $\mathbb{N}_{l}:=\{l, l+1, \ldots\}, \mathbb{N}:=\mathbb{N}_{1}, k \in \mathbb{N}_{2}$, and let $\mathcal{H}(k)$ denote the class of function $f \in \mathcal{H}$ of the form (1.3) for which, $f_{z}^{\prime}(0)=1$ and the coefficients $a_{0}, a_{2}, \ldots, a_{k-1}, b_{1}, \ldots, b_{k-1}$ vanish i.e.

$$
f(z)=z+\sum_{n=k}^{\infty}\left(a_{n} z^{n}+\overline{b_{n} z^{n}}\right) \quad(z \in \mathbb{U}) .
$$

Then we say that $f$ is the function with missing coefficients. Moreover, let $\mathcal{S}_{\mathcal{H}}(k)$ denote the class of function $f \in \mathcal{H}(k)$ which are univalent in $\mathbb{U}$.

Email addresses: jdziok@ur.edu.pl

Received: 13.04.2018; Accepted: 24.07.2018 
Now, we define the convolution of functions $f_{1}$ and $f_{2}$ of the form

$$
f_{l}(z)=z+\sum_{n=k}^{\infty} a_{l, n} z^{n}+\sum_{n=k}^{\infty} \overline{b_{l, n} z^{n}} \quad(z \in \mathbb{U}, l \in \mathbb{N})
$$

by

$$
\left(f_{1} * f_{2}\right)(z)=z+\sum_{n=k}^{\infty} a_{1, n} a_{2, n} z^{n}+\sum_{n=k}^{\infty} \overline{b_{1, n} b_{2, n} z^{n}} \quad(z \in \mathbb{U}) .
$$

We say that a function $f \in \mathcal{S}_{\mathcal{H}}(2)$ is harmonic starlike in $\mathbb{U}(r):=\{z \in \mathbb{C}:|z|<r\}$ if

$$
\frac{\partial}{\partial t}\left(\arg f\left(r e^{i t}\right)\right)>0 \quad(0 \leq t \leq 2 \pi)
$$

i.e. $f$ maps the circle $\partial \mathbb{U}(r)$ onto a closed curve that is starlike with respect to the origin. It is easy to verify, that the above condition is equivalent to the following

$$
\operatorname{Re} \frac{D_{\mathcal{H}} f(z)}{f(z)}>0 \quad(|z|=r)
$$

where

$$
D_{\mathcal{H}} f(z):=z h^{\prime}(z)-\overline{z g^{\prime}(z)} \quad(z \in \mathbb{U}) .
$$

Ruscheweyh [20] introduced on the class of analytic function an operator $\mathcal{D}^{\lambda}$ defined by the convolution:

which implies that

$$
\mathcal{D}^{\lambda} f(z)=\frac{z}{(1-z)^{\lambda+1}} * f(z) \quad(\lambda \neq-1),
$$

$$
\mathcal{D}^{n} f(z)=\frac{z\left(z^{n-1} f(z)\right)^{(n)}}{n !} \quad\left(n \in \mathbb{N}_{0}\right) .
$$

Let $D_{\mathcal{H}}^{\lambda, \tau}: \mathcal{H} \rightarrow \mathcal{H}$ denote the linear operator defined for a function $f=h+\bar{g} \in \mathcal{H}$ by $($ see $[5])$

$$
D_{\mathcal{H}}^{\lambda, \tau} f:=\mathcal{D}^{\lambda} h+\tau \overline{\mathcal{D}^{\lambda} g} \quad(|\tau|=1) .
$$

The operator $D_{\mathcal{H}}^{\lambda, \tau}$ for $\tau=(-1)^{n}$ was investigated in [17] (see also [7, 10,22]).

We say that a function $f \in \mathcal{H}$ is subordinate to a function $F \in \mathcal{H}$, and write $f(z) \prec F(z)$ (or simply $f \prec F$ ) if there exists a complex-valued function wwhich maps $\mathbb{U}$ into oneself with $\omega(0)=0$, such that $f(z)=F(\omega(z))(z \in \mathbb{U})$.

Let $A, B$ be complex parameters, $A \neq B$. We denote by $\mathcal{S}_{\mathcal{H}}^{\lambda, \tau}(k ; A, B)$ the class of functions $f \in \mathcal{S}_{\mathcal{H}}(k)$ such that

$$
\frac{D_{\mathcal{H}}\left(D_{\mathcal{H}}^{\lambda, \tau} f\right)(z)}{D_{\mathcal{H}}^{\lambda, \tau} f(z)} \prec \frac{1+A z}{1+B z} .
$$

Also, by $\mathcal{S}_{\mathcal{H}}^{\lambda, \tau}(k ; A, B)$ we denote the class of functions $f \in \mathcal{S}_{\mathcal{H}}(k)$ such that

$$
\frac{D_{\mathcal{H}}^{\lambda, \tau} f(z)}{z} \prec \frac{1+A z}{1+B z} .
$$

In particular, for $k=2$ we obtain the classes studied in [5]. If we put $\lambda=n \in \mathbb{N}_{0}$, $\tau=(-1)^{n}$, then we obtain the classes

$$
\mathcal{S}_{\mathcal{H}}^{n}(k ; A, B):=\mathcal{S}_{\mathcal{H}}^{n,(-1)^{n}}(k ; A, B), \mathcal{R}_{\mathcal{H}}^{n}(k ; A, B):=\mathcal{R}_{\mathcal{H}}^{n,(-1)^{n}}(k ; A, B)
$$

related to the harmonic Ruscheweyh derivatives $\mathcal{D}_{\mathcal{H}}^{n} f$ (see [7]). The classes $\mathcal{S}_{\mathcal{H}}(k ; A, B):=$ $\mathcal{S}_{\mathcal{H}}^{0}(k ; A, B), \mathcal{K}_{\mathcal{H}}(k ; A, B):=\mathcal{S}_{\mathcal{H}}^{1}(k ; A, B)$ and $\mathcal{R}_{\mathcal{H}}(k ; A, B):=\mathcal{R}_{\mathcal{H}}^{1}(k ; A, B)$ are defined in [4] with restrictions $-B \leq A<B \leq 1, k=2$.

In this paper we obtain some necessary and sufficient conditions for defined classes of functions. Some topological properties and extreme points of the classes are also considered. By using extreme points theory we obtain coefficients estimates, distortion theorems, 
integral mean inequalities for these classes of functions. Some applications of the main results are also considered.

\section{Dual sets}

Let $\mathcal{V} \subset \mathcal{H}, \mathbb{U}_{0}:=\mathbb{U} \backslash\{0\}$. Due to Ruscheweyh [19] we define the dual set of $\mathcal{V}$ by

$$
\mathcal{V}^{*}:=\left\{f \in \mathcal{S}_{\mathcal{H}}(k): \bigwedge_{q \in \mathcal{V}}(f * q)(z) \neq 0 \quad\left(z \in \mathbb{U}_{0}\right)\right\} .
$$

The object of this section is to show that the defined classes of functions can be presented as dual sets.

\section{Theorem 2.1.}

where

$$
\mathcal{S}_{\mathcal{H}}^{\lambda, \tau}(k ; A, B)=\left\{\psi_{\xi}:|\xi|=1\right\}^{*}
$$

$$
\begin{aligned}
\psi_{\xi}(z): & =z \frac{(B-A) \xi+(1+\lambda+\lambda B \xi+A \xi) z}{(1-z)^{\lambda+2}} \\
& -\tau \bar{z} \frac{2+(A+B) \xi-(1-\lambda-\lambda B \xi+A \xi) \bar{z}}{(1-\bar{z})^{\lambda+2}} \quad(z \in \mathbb{U}) .
\end{aligned}
$$

Proof. Let $f \in \mathcal{H}$ be of the form (1.1). Then $f \in \mathcal{S}_{\mathcal{H}}^{\lambda, \tau}(k ; A, B)$ if and only if it satisfies (1.7) or equivalently

$$
\frac{D_{\mathcal{H}}\left(D_{\mathcal{H}}^{\lambda, \tau} f\right)(z)}{D_{\mathcal{H}}^{\lambda, \tau} f(z)} \neq \frac{1+A \xi}{1+B \xi} \quad\left(z \in \mathbb{U}_{0},|\xi|=1\right) .
$$

Since

$$
D_{\mathcal{H}}\left(D_{\mathcal{H}}^{\lambda, \tau} h\right)(z)=h(z) * \frac{z}{(1-z)^{\lambda+1}} * \frac{z}{(1-z)^{2}}=h(z) * \frac{z+\lambda z^{2}}{(1-z)^{\lambda+2}},
$$

from (2.2) it follows that

$$
\begin{aligned}
& (1+B \xi) D_{\mathcal{H}}\left(D_{\mathcal{H}}^{\lambda, \tau} f\right)(z)-(1+A \xi) D_{\mathcal{H}}^{\lambda, \tau} f(z) \\
= & (1+B \xi) D_{\mathcal{H}}\left(D_{\mathcal{H}}^{\lambda, \tau} h\right)(z)-(1+A \xi) D_{\mathcal{H}}^{\lambda, \tau} h(z) \\
& -\tau\left[(1+B \xi) \overline{D_{\mathcal{H}}\left(D_{\mathcal{H}}^{\lambda, \tau} g\right)(z)}+(1+A \xi) \overline{D_{\mathcal{H}}^{\lambda, \tau} h(z)}\right] \\
= & h(z) *\left(\frac{(1+B \xi)(1+\lambda z) z}{(1-z)^{\lambda+2}}-\frac{(1+A \xi) z}{(1-z)^{\lambda+1}}\right) \\
& -\tau \overline{g(z)} *\left(\frac{(1+B \xi)(1+\bar{\lambda} \bar{z}) \bar{z}}{(1-\bar{z})^{\lambda+2}}+\frac{(1+A \xi) \bar{z}}{(1-\bar{z})^{\lambda+1}}\right) \\
= & f(z) * \psi_{\xi}(z) \neq 0 \quad\left(z \in \mathbb{U}_{0},|\xi|=1\right) .
\end{aligned}
$$

Thus, $f \in \mathcal{S}_{\mathcal{H}}^{\lambda, \tau}(k ; A, B)$ if and only if $f(z) * \psi_{\xi}(z) \neq 0$ for $z \in \mathbb{U}_{0},|\xi|=1$ i.e. $\mathcal{S}_{\mathcal{H}}^{\lambda, \tau}(k ; A, B)=\left\{\psi_{\xi}:|\xi|=1\right\}^{*}$.

Similarly as Theorem 2.1 we prove the following theorem.

\section{Theorem 2.2.}

$$
\mathcal{R}_{\mathcal{H}}^{\lambda, \tau}(k ; A, B)=\left\{\delta_{\xi}:|\xi|=1\right\}^{*},
$$

where

$$
\delta_{\xi}(z):=z \frac{1+B \xi-(1+A \xi)(1-z)^{\lambda+1}}{(1-z)^{\lambda+1}}+\tau \bar{z} \frac{1+B \xi}{(1-\bar{z})^{\lambda+1}} \quad(z \in \mathbb{U}) .
$$


If we put $\lambda=n \in \mathbb{N}_{0}, \tau=(-1)^{n}$, in Theorems 2.1 and 2.2 we obtain the following results.

\section{Theorem 2.3.}

$$
\mathcal{S}_{\mathcal{H}}^{n}(k ; A, B)=\left\{\rho_{\xi}:|\xi|=1\right\}^{*},
$$

where

$$
\begin{aligned}
\rho_{\xi}(z)= & z \frac{(B-A) \xi+(1+n+(n B+A) \xi) z}{(1-z)^{n+2}} \\
& -(-1)^{n} \bar{z} \frac{2+(A+B) \xi+(n-1+(n B-A) \xi) \bar{z}}{(1-\bar{z})^{n+2}} \quad(z \in \mathbb{U}) .
\end{aligned}
$$

Theorem 2.4.

$$
\mathcal{R}_{\mathcal{H}}^{n}(k ; A, B)=\left\{\delta_{\xi}:|\xi|=1\right\}^{*},
$$

where

$$
\delta_{\xi}(z):=z \frac{1+B \xi-(1+A \xi)(1-z)^{n+1}}{(1-z)^{n+1}}+(-1)^{n} \bar{z} \frac{1+B \xi}{(1-\bar{z})^{n+1}} \quad(z \in \mathbb{U}) .
$$

Moreover, if we get $n=0$ and $n=1$ in Theorem 2.3 and $n=1$ in Theorem 2.4 we obtain the following results.

\section{Theorem 2.5.}

$$
\mathcal{S}_{\mathcal{H}}(k ; A, B)=\left\{\psi_{\xi}:|\xi|=1\right\}^{*},
$$

where

$$
\psi_{\xi}(z):=z \frac{(B-A) \xi+(1+A \xi) z}{(1-z)^{2}}-\bar{z} \frac{2+(A+B) \xi-(1+A \xi) \bar{z}}{(1-\bar{z})^{2}} \quad(z \in \mathbb{U}) .
$$

Theorem 2.6.

$$
\mathcal{K}_{\mathcal{H}}(k ; A, B)=\left\{\psi_{\xi}:|\xi|=1\right\}^{*}
$$

where

$$
\psi_{\xi}(z):=z \frac{(B-A) \xi+(2+A \xi+B \xi) z}{(1-z)^{3}}-\bar{z} \frac{2+(A+B) \xi+(B-A) \xi \bar{z}}{(1-\bar{z})^{3}} \quad(z \in \mathbb{U}) .
$$

\section{Correlated coefficients}

Let us consider the complex-value function $\varphi$ of the form

$$
\varphi=u+\bar{v}, \quad u(z)=\sum_{n=0}^{\infty} u_{n} z^{n}, \quad v(z)=\sum_{n=1}^{\infty} v_{n} z^{n} \quad(z \in \mathbb{U}),
$$

where $u, v$ are functions analytic in $\mathbb{U}$.

We say that a function $f \in \mathcal{H}$ of the form (1.4) has correlated coefficients with respect to the function $\varphi$, if

$$
u_{n} a_{n}=-\left|u_{n}\right|\left|a_{n}\right|, v_{n} b_{n}=\left|v_{n}\right|\left|b_{n}\right| \quad\left(n \in \mathbb{N}_{k}\right) .
$$

In particular, if there exists a real number $\eta$ such that

$$
\varphi(z)=\frac{z}{1-e^{i \eta} z}+\frac{\bar{z} e^{-2 i \eta}}{1-e^{-i \eta \bar{z}}}=\sum_{n=1}^{\infty} e^{i(n-1) \eta} z^{n}+e^{-i(n+1) \eta} \bar{z}^{n} \quad(z \in \mathbb{U}),
$$

then we obtain functions with varying coefficients defined by Jahangiri and Silverman [11] (see also [6]). Moreover, if we take $\eta=0$ i.e.

$$
\varphi(z)=2 \operatorname{Re} \frac{z}{1-z}=\sum_{n=1}^{\infty}\left(z^{n}+\bar{z}^{n}\right) \quad(z \in \mathbb{U})
$$


then we obtain functions with negative coefficients introduced by Silverman [21]. These functions were intensively investigated by many authors (for example, see $[3-5,7,9,11,13$, 24]).

Let $\mathcal{T}^{\lambda, \tau}(k, \eta)$ denote the class of functions $f \in \mathcal{H}$ of the correlated coefficients with respect to the function

$$
\varphi(z):=\frac{z}{\left(1-e^{i \eta} z\right)^{\lambda+1}}+\tau \frac{\bar{z} e^{-2 i \eta}}{\left(1-e^{-i \eta} \bar{z}\right)^{\bar{\lambda}+1}} \quad(z \in \mathbb{U}) .
$$

Moreover, let us define

$\mathcal{S}_{\mathcal{T}}^{\lambda, \tau}(k, \eta ; A, B):=\mathcal{T}^{\lambda, \tau}(k, \eta) \cap \mathcal{S}_{\mathcal{H}}^{\lambda, \tau}(k ; A, B), \mathcal{R}_{\mathcal{T}}^{\lambda, \tau}(k, \eta ; A, B):=\mathcal{T}^{\lambda, \tau}(k, \eta) \cap \mathcal{R}_{\mathcal{H}}^{\lambda, \tau}(k ; A, B)$, where $\eta ; A, B$ are real parameters, $B>\max \{0, A\}$.

Let $f \in \mathcal{H}$ be of the form (1.4). Thus, by (1.6) and (3.3) we have

$$
D_{\mathcal{H}}^{\lambda, \tau} f(z)=z+\sum_{n=k}^{\infty} \lambda_{n} a_{n} z^{n}+\tau \sum_{n=k}^{\infty} \overline{\lambda_{n} b_{n}} \bar{z}^{n} \quad(z \in \mathbb{U})
$$

and

where

$$
\varphi(z)=\sum_{n=1}^{\infty} \lambda_{n} e^{i(n-1) \eta} z^{n}+\tau \sum_{n=1}^{\infty} \overline{\lambda_{n}} e^{-i(n+1) \eta} \bar{z}^{n} \quad(z \in \mathbb{U})
$$

$$
\lambda_{1}:=1, \quad \lambda_{n}:=\frac{(\lambda+1) \cdot \ldots \cdot(\lambda+n-1)}{(n-1) !} \quad\left(n \in \mathbb{N}_{2}\right) .
$$

Moreover, let us assume

$$
\left|\lambda_{n}\right| \geq\left|\lambda_{k}\right| \geq 1, B>\max \{0, A\} .
$$

Theorem 3.1. If a function $f \in \mathcal{H}$ of the form (1.4) satisfies the condition

$$
\sum_{n=k}^{\infty}\left(\left|\alpha_{n}\right|\left|a_{n}\right|+\left|\beta_{n}\right|\left|b_{n}\right|\right) \leq B-A
$$

where

$$
\alpha_{n}=\lambda_{n}\{n(1+B)-(1+A)\}, \quad \beta_{n}=\lambda_{n}\{n(1+B)+(1+A)\},
$$

then $f \in \mathcal{S}_{\mathcal{H}}^{\lambda, \tau}(k ; A, B)$.

Proof. It is clear that the theorem is true for the function $f(z) \equiv z$. Let $f \in \mathcal{H}(k)$ be a function of the form (1.4) and let there exist $n \in \mathbb{N}_{k}$ such that $a_{n} \neq 0$ or $b_{n} \neq 0$. By (3.6) we have

Thus, by (3.7) we get

$$
\frac{\left|\alpha_{n}\right|}{B-A} \geq n, \frac{\left|\beta_{n}\right|}{B-A} \geq n, \quad n \in \mathbb{N}_{k} .
$$

$$
\sum_{n=k}^{\infty}\left(n\left|a_{n}\right|+n\left|b_{n}\right|\right) \leq 1
$$

and

$$
\begin{aligned}
\left|h^{\prime}(z)\right|-\left|g^{\prime}(z)\right| & \geq 1-\sum_{n=k}^{\infty} n\left|a_{n}\right||z|^{n}-\sum_{n=k}^{\infty} n\left|b_{n}\right||z|^{n} \geq 1-|z| \sum_{n=k}^{\infty}\left(n\left|a_{n}\right|+n\left|b_{n}\right|\right) \\
& \geq 1-\frac{|z|}{B-A} \sum_{n=k}^{\infty}\left(\left|\alpha_{n}\right|\left|a_{n}\right|+\left|\beta_{n}\right|\left|b_{n}\right|\right) \geq 1-|z|>0 \quad(z \in \mathbb{U}) .
\end{aligned}
$$

Therefore, by (1.2) the function $f$ is locally univalent and sense-preserving in $\mathbb{U}$. Moreover, if $z_{1}, z_{2} \in \mathbb{U}, z_{1} \neq z_{2}$, then

$$
\left|\frac{z_{1}^{n}-z_{2}^{n}}{z_{1}-z_{2}}\right|=\left|\sum_{l=1}^{n} z_{1}^{l-1} z_{2}^{n-l}\right| \leq \sum_{l=1}^{n}\left|z_{1}\right|^{l-1}\left|z_{2}\right|^{n-l}<n \quad\left(n \in \mathbb{N}_{k}\right) .
$$


Hence, by (3.10) we have

$$
\begin{aligned}
\left|f\left(z_{1}\right)-f\left(z_{2}\right)\right| & \geq\left|h\left(z_{1}\right)-h\left(z_{2}\right)\right|-\left|g\left(z_{1}\right)-g\left(z_{2}\right)\right| \\
& =\left|z_{1}-z_{2}-\sum_{n=k}^{\infty} a_{n}\left(z_{1}^{n}-z_{2}^{n}\right)\right|-\left|\sum_{n=k}^{\infty} \overline{b_{n}\left(z_{1}^{n}-z_{2}^{n}\right)}\right| \\
& \geq\left|z_{1}-z_{2}\right|-\sum_{n=k}^{\infty}\left|a_{n}\right|\left|z_{1}^{n}-z_{2}^{n}\right|-\sum_{n=k}^{\infty}\left|b_{n}\right|\left|z_{1}^{n}-z_{2}^{n}\right| \\
& =\left|z_{1}-z_{2}\right|\left(1-\sum_{n=k}^{\infty}\left|a_{n}\right|\left|\frac{z_{1}^{n}-z_{2}^{n}}{z_{1}-z_{2}}\right|-\sum_{n=k}^{\infty}\left|b_{n}\right|\left|\frac{z_{1}^{n}-z_{2}^{n}}{z_{1}-z_{2}}\right|\right) \\
& >\left|z_{1}-z_{2}\right|\left(1-\sum_{n=k}^{\infty} n\left|a_{n}\right|-\sum_{n=k}^{\infty} n\left|b_{n}\right|\right) \geq 0 .
\end{aligned}
$$

This leads to the univalence of $f$ i.e. $f \in \mathcal{S}_{\mathcal{H}}$. Therefore, $f \in \mathcal{S}_{\mathcal{T}}^{\lambda, \tau}(k ; A, B)$ if and only if there exists a complex-valued function $\omega, \omega(0)=0,|\omega(z)|<1(z \in \mathbb{U})$ such that

$$
\frac{D_{\mathcal{H}}\left(D_{\mathcal{H}}^{\lambda, \tau} f\right)(z)}{D_{\mathcal{H}}^{\lambda, \tau} f(z)}=\frac{1+A \omega(z)}{1+B \omega(z)} \quad(z \in \mathbb{U}),
$$

or equivalently

$$
\left|\frac{D_{\mathcal{H}}\left(D_{\mathcal{H}}^{\lambda \tau} f\right)(z)-D_{\mathcal{H}}^{\lambda \tau} f(z)}{B D_{\mathcal{H}}\left(D_{\mathcal{H}}^{\lambda \tau} f\right)(z)-A\left(D_{\mathcal{H}}^{\lambda \tau} f(z)\right)(z)}\right|<1 \quad(z \in \mathbb{U}) .
$$

Thus, it is suffice to prove that

$$
\left|D_{\mathcal{H}}\left(D_{\mathcal{H}}^{\lambda \tau} f\right)(z)-D_{\mathcal{H}}^{\lambda \tau} f(z)\right|-\left|B D_{\mathcal{H}}\left(D_{\mathcal{H}}^{\lambda \tau} f\right)(z)-A D_{\mathcal{H}}^{\lambda, \tau} f(z)\right|<0 \quad(z \in \mathbb{U} \backslash\{0\}) .
$$

Indeed, letting $|z|=r(0<r<1)$ we have

$$
\begin{aligned}
& \left|D_{\mathcal{H}}\left(D_{\mathcal{H}}^{\lambda \tau} f\right)(z)-D_{\mathcal{H}}^{\lambda \tau} f(z)\right|-\left|B D_{\mathcal{H}}\left(D_{\mathcal{H}}^{\lambda \tau} f\right)(z)-A D_{\mathcal{H}}^{\lambda, \tau} f(z)\right| \\
& =\left|\sum_{n=k}^{\infty}(n-1) \lambda_{n} a_{n} z^{n}-\sum_{n=k}^{\infty}(n+1) \tau \overline{\lambda_{n} b_{n}} \bar{z}^{n}\right| \\
& -\left|(B-A) z+\sum_{n=k}^{\infty}(B n-A) \lambda_{n} a_{n} z^{n}+\sum_{n=k}^{\infty}(B n+A) \tau \overline{\lambda_{n} b_{n}} \bar{z}^{n}\right| \\
& \leq \sum_{n=k}^{\infty}(n-1)\left|\lambda_{n} a_{n}\right| r^{n}+\sum_{n=k}^{\infty}(n+1)\left|\lambda_{n} b_{n}\right| r^{n}-(B-A) r \\
& +\sum_{n=k}^{\infty}(B n-A)\left|\lambda_{n} a_{n}\right| r^{n}+\sum_{n=k}^{\infty}(B n+A)\left|\lambda_{n} b_{n}\right| r^{n} \\
& \leq r\left\{\sum_{n=k}^{\infty}\left(\left|\alpha_{n}\right|\left|a_{n}\right|+\left|\beta_{n}\right|\left|b_{n}\right|\right) r^{n-1}-(B-A)\right\}<0 .
\end{aligned}
$$

whence $f \in \mathcal{S}_{\mathcal{H}}^{\lambda, \tau}(k ; A, B)$.

The next theorem, shows that the condition (3.7) is also the sufficient condition for a function $f \in \mathcal{H}$ of correlated coefficients to be in the class $\mathcal{S}_{\mathcal{T}}^{\lambda, \tau}(k, \eta ; A, B)$.

Theorem 3.2. Let $f \in \mathcal{T}^{\lambda, \tau}(k, \eta)$ be a function of the form (1.4). Then $f \in \mathcal{S}_{\mathcal{T}}^{\lambda, \tau}(k, \eta ; A, B)$ if and only if the condition (3.7) holds true. 
Proof. In view of Theorem 3.1 we need only to show that each function $f \in \mathcal{S}_{\mathcal{T}}^{\lambda, \tau}(k, \eta ; A, B)$ satisfies the coefficient inequality (3.7). If $f \in \mathcal{S}_{\mathcal{T}}^{\lambda, \tau}(k, \eta ; A, B)$, then it is of the form (1.4) with (3.2) and it satisfies (3.11) or equivalently

$$
\left|\frac{\sum_{n=k}^{\infty}(n-1) \lambda_{n} a_{n} z^{n}-(n+1) \tau \overline{\lambda_{n} b_{n}} \bar{z}^{n}}{(B-A) z+\sum_{n=k}^{\infty}\left\{(B n-A) \lambda_{n} a_{n} z^{n}-(B n+A) \tau \overline{\lambda_{n} b_{n}} \bar{z}^{n}\right\}}\right|<1 \quad(z \in \mathbb{U}) .
$$

Since $f \in \mathcal{T}^{\lambda, \tau}(k, \eta)$, by (3.4) and (3.2) we have

$$
\lambda_{n} a_{n} e^{i(n-1) \eta}=-\left|\lambda_{n}\right|\left|a_{n}\right|, \tau \overline{\lambda_{n} b_{n}} e^{-i(n+1) \eta}=\left|\lambda_{n}\right|\left|b_{n}\right| \quad\left(n \in \mathbb{N}_{k}\right) .
$$

Therefore, putting $z=r e^{i \eta}(0 \leq r<1)$ in $(3.12)$ we obtain

$$
\frac{\sum_{n=k}^{\infty}(n-1)\left|\lambda_{n}\right|\left|a_{n}\right|+(n+1)\left|\lambda_{n}\right|\left|b_{n}\right| r^{n-1}}{(B-A)-\sum_{n=k}^{\infty}\left\{(B n-A)\left|\lambda_{n}\right|\left|a_{n}\right|+(B n+A)\left|\lambda_{n}\right|\left|b_{n}\right|\right\} r^{n-1}}<1 .
$$

It is clear that the denominator of the left hand side cannot vanish for $r \in\langle 0,1)$. Moreover, it is positive for $r=0$, and in consequence for $r \in\langle 0,1)$. Thus, by (3.13) we have

$$
\sum_{n=k}^{\infty}\left(\left|\alpha_{n}\right|\left|a_{n}\right|+\left|\beta_{n}\right|\left|b_{n}\right|\right) r^{n-1}<B-A \quad(0 \leq r<1) .
$$

The sequence of partial sums $\left\{S_{n}\right\}$ associated with the series $\sum_{n=k}^{\infty}\left(\left|\alpha_{n}\right|\left|a_{n}\right|+\left|\beta_{n}\right|\left|b_{n}\right|\right)$ is non-decreasing sequence. Moreover, by (3.14) it is bounded by $B-A$. Hence, the sequence $\left\{S_{n}\right\}$ is convergent and

$$
\sum_{n=k}^{\infty}\left(\left|\alpha_{n}\right|\left|a_{n}\right|+\left|\beta_{n}\right|\left|b_{n}\right|\right)=\lim _{n \rightarrow \infty} S_{n} \leq B-A,
$$

which yields the assertion (3.7).

The following result may be proved in much the same way as Theorem 3.2.

Theorem 3.3. Let $f \in \mathcal{H}$ be a function of the form (1.4). Then $f \in \mathcal{R}_{\mathcal{T}}^{\lambda, \tau}(k, \eta ; A, B)$ if and only if

$$
\sum_{n=k}^{\infty}\left|\lambda_{n}\right|\left(\left|a_{n}\right|+\left|b_{n}\right|\right) \leq \frac{B-A}{1+B}
$$

By Theorems 3.2 and 3.3 we have the following corollary.

Corollary 3.4. Let $a=\frac{1+A}{1+B}$ and

$$
\begin{aligned}
& \phi(z)=z+\sum_{n=k}^{\infty}\left(\frac{1}{n-a} z^{n}+\frac{1}{n+a} \bar{z}^{n}\right) \quad(z \in \mathbb{U}), \\
& \omega(z)=z+\sum_{n=k}^{\infty}\left((n-a) z^{n}+(n+a) \bar{z}^{n}\right) \quad(z \in \mathbb{U}) .
\end{aligned}
$$

Then

$$
\begin{aligned}
& f \in \mathcal{R}_{\mathcal{T}}^{\lambda, \tau}(k, \eta ; A, B) \Leftrightarrow f * \phi \in \mathcal{S}_{\mathcal{T}}^{\lambda, \tau}(k, \eta ; A, B), \\
& f \in \mathcal{S}_{\mathcal{T}}^{\lambda, \tau}(k, \eta ; A, B) \Leftrightarrow f * \omega \in \mathcal{R}_{\mathcal{T}}^{\lambda, \tau}(k, \eta ; A, B) .
\end{aligned}
$$

In particular,

$$
\mathcal{R}_{\mathcal{T}}^{1}(k, \eta ;-1, B)=\mathcal{S}_{\mathcal{T}}^{0}(k, \eta ;-1, B) .
$$




\section{Extreme points}

We consider the usual topology on $\mathcal{H}$ defined by a metric in which a sequence $\left\{f_{n}\right\}$ in $\mathcal{H}$ converges to $f$ if and only if it converges to $f$ uniformly on each compact subset of $\mathbb{U}$. It follows from the theorems of Weierstrass and Montel that this topological space is complete.

Let $\mathcal{F}$ be a subclass of the class $\mathcal{H}$. A functions $f \in \mathcal{F}$ is called an extreme point of $\mathcal{F}$ if the condition

$$
f=\gamma f_{1}+(1-\gamma) f_{2} \quad\left(f_{1}, f_{2} \in \mathcal{F}, 0<\gamma<1\right)
$$

implies $f_{1}=f_{2}=f$. We shall use the notation $E \mathcal{F}$ to denote the set of all extreme points of $\mathcal{F}$. It is clear that $E \mathcal{F} \subset \mathcal{F}$.

We say that $\mathcal{F}$ is locally uniformly bounded if for each $r, 0<r<1$, there is a real constant $M=M(r)$ so that

$$
|f(z)| \leq M \quad(f \in \mathcal{F},|z| \leq r) .
$$

We say that a class $\mathcal{F}$ is convex if

$$
\gamma f+(1-\gamma) g \in \mathcal{F} \quad(f, g \in \mathcal{F}, 0 \leq \gamma \leq 1) .
$$

Moreover, we define the closed convex hull of $\mathcal{F}$ as the intersection of all closed convex subsets of $\mathcal{H}$ that contain $\mathcal{F}$. We denote the closed convex hull of $\mathcal{F}$ by $\overline{c o} \mathcal{F}$.

A real-valued functional $\mathcal{J}: \mathcal{H} \rightarrow \mathbb{R}$ is called convex on a convex class $\mathcal{F} \subset \mathcal{H}$ if

$$
\mathcal{J}(\gamma f+(1-\gamma) g) \leq \gamma \mathcal{J}(f)+(1-\gamma) \mathcal{J}(g) \quad(f, g \in \mathcal{F}, 0 \leq \gamma \leq 1) .
$$

The Krein-Milman theorem (see [14]) is fundamental in the theory of extreme points. In particular, it implies the following lemma (due to [8]).

Lemma 4.1. [4, pp.45] Let $\mathcal{F}$ be a non-empty compact convex subclass of the class $\mathcal{H}$ and $\mathcal{J}: \mathcal{H} \rightarrow \mathbb{R}$ be a real-valued, continuous and convex functional on $\mathcal{F}$. Then

$$
\max \{\mathcal{J}(f): f \in \mathcal{F}\}=\max \{\mathcal{J}(f): f \in E \mathcal{F}\} .
$$

Since $\mathcal{H}$ is a complete metric space, Montel's theorem (see [16]) implies the following lemma.

Lemma 4.2. A class $\mathcal{F} \subset \mathcal{H}$ is compact if and only if $\mathcal{F}$ is closed and locally uniformly bounded.

Theorem 4.3. The class $\mathcal{S}_{\mathcal{T}}^{\lambda, \tau}(k, \eta ; A, B)$ is convex and compact subset of $\mathcal{H}$.

Proof. Let $f_{1}, f_{2} \in \mathcal{S}_{\mathcal{T}}^{\lambda, \tau}(k, \eta ; A, B)$ be functions of the form (1.5), $0 \leq \gamma \leq 1$. Since

$$
\gamma f_{1}(z)+(1-\gamma) f_{2}(z)=z+\sum_{n=k}^{\infty}\left\{\left(\gamma a_{1, n}+(1-\gamma) a_{2, n}\right) z^{n}+\overline{\left(\gamma b_{1, n}+(1-\gamma) b_{2, n}\right) z^{n}}\right\},
$$

and by Theorem 3.2 we have

$$
\begin{aligned}
& \sum_{n=k}^{\infty}\left\{\left|\alpha_{n}\right|\left|\gamma a_{1, n}+(1-\gamma) a_{2, n}\right|+\left|\beta_{n}\right|\left|\gamma b_{1, n}+(1-\gamma) b_{2, n} z^{n}\right|\right\} \\
\leq & \gamma \sum_{n=k}^{\infty}\left\{\left|\alpha_{n} a_{1, n}\right|+\left|\beta_{n} b_{1, n}\right|\right\}+(1-\gamma) \sum_{n=k}^{\infty}\left\{\left|\alpha_{n} a_{2, n}\right|+\left|\beta_{n} b_{2, n}\right|\right\} \\
\leq & \gamma(B-A)+(1-\gamma)(B-A)=B-A,
\end{aligned}
$$

the function $\phi=\gamma f_{1}+(1-\gamma) f_{2}$ belongs to the class $\mathcal{S}_{\mathcal{T}}^{\lambda, \tau}(k, \eta ; A, B)$. Hence, the class is convex. Furthermore, for $f \in \mathcal{S}_{\mathcal{T}}^{\lambda, \tau}(k, \eta ; A, B),|z| \leq r, 0<r<1$, we have

$$
|f(z)| \leq r+\sum_{n=k}^{\infty}\left(\left|a_{n}\right|+\left|b_{n}\right|\right) r^{n} \leq r+\sum_{n=k}^{\infty}\left(\left|\alpha_{n}\right|\left|a_{n}\right|+\left|\beta_{n}\right|\left|b_{n}\right|\right) \leq r+(B-A) .
$$


Thus, we conclude that the class $\mathcal{S}_{\mathcal{T}}^{\lambda, \tau}(k, \eta ; A, B)$ is locally uniformly bounded. By Lemma 4.2, we only need to show that it is closed i.e. if $f_{l} \in \mathcal{S}_{\mathcal{T}}^{\lambda, \tau}(k, \eta ; A, B) \quad(l \in \mathbb{N})$ and $f_{l} \rightarrow f$, then $f \in \mathcal{S}_{\mathcal{T}}^{\lambda, \tau}(k, \eta ; A, B)$. Let $f_{l}$ and $f$ be given by (1.5) and (1.4), respectively. Using Theorem 3.2 we have

$$
\sum_{n=k}^{\infty}\left(\left|\alpha_{n} a_{l, n}\right|+\left|\beta_{n} b_{l, n}\right|\right) \leq B-A \quad(l \in \mathbb{N}) .
$$

Since $f_{l} \rightarrow f$, we conclude that $\left|a_{l, n}\right| \rightarrow\left|a_{n}\right|$ and $\left|b_{l, n}\right| \rightarrow\left|b_{n}\right|$ as $l \rightarrow \infty \quad(n \in \mathbb{N})$. The sequence of partial sums $\left\{S_{n}\right\}$ associated with the series $\sum_{n=k}^{\infty}\left(\left|\alpha_{n} a_{n}\right|+\left|\beta_{n} b_{n}\right|\right)$ is non-decreasing sequence. Moreover, by (4.2) it is bounded by $B-A$. Therefore, the sequence $\left\{S_{n}\right\}$ is convergent and

$$
\sum_{n=k}^{\infty}\left(\left|\alpha_{n} a_{n}\right|+\left|\beta_{n} b_{n}\right|\right)=\lim _{n \rightarrow \infty} S_{n} \leq B-A .
$$

This gives the condition (3.7), and, in consequence, $f \in \mathcal{S}_{\mathcal{T}}^{\lambda, \tau}(k, \eta ; A, B)$, which completes the proof.

Theorem 4.4.

$$
\operatorname{ES}_{\mathcal{T}}^{\lambda, \tau}(k, \eta ; A, B)=\left\{h_{n}: n \in \mathbb{N}_{k-1}\right\} \cup\left\{g_{n}: n \in \mathbb{N}_{k}\right\}
$$

where

$$
h_{k-1}(z)=z, h_{n}(z)=z-\frac{B-A}{\alpha_{n} e^{i(n-1) \eta}} z^{n}, g_{n}(z)=z+\frac{\bar{\tau}(B-A)}{\overline{\beta_{n}} e^{-i(n+1) \eta}} \bar{z}^{n} \quad(z \in \mathbb{U}) .
$$

Proof. Suppose that $0<\gamma<1$ and

$$
g_{n}=\gamma f_{1}+(1-\gamma) f_{2},
$$

where $f_{1}, f_{2} \in \mathcal{S}_{\mathcal{T}}^{\lambda, \tau}(k, \eta ; A, B)$ are functions of the form (1.5). Then, by (3.7) we have $\left|b_{1, n}\right|=\left|b_{2, n}\right|=\frac{B-A}{\left|\beta_{n}\right|}$, and, in consequence, $a_{1, l}=a_{2, l}=0$ for $l \in \mathbb{N}_{k}$ and $b_{1, l}=b_{2, l}=0$ for $l \in \mathbb{N}_{k} \backslash\{n\}$. It follows that $g_{n}=f_{1}=f_{2}$, and consequently $g_{n} \in E \mathcal{S}_{\mathcal{T}}^{*}(k, \eta ; A, B)$. Similarly, we verify that the functions $h_{n}$ of the form (4.3) are the extreme points of the class $\mathcal{S}_{\mathcal{T}}^{\lambda, \tau}(k, \eta ; A, B)$. Now, suppose that a function $f$ belongs to the set $E \mathcal{S}_{\mathcal{T}}^{\lambda, \tau}(k, \eta ; A, B)$ and $f$ is not of the form (4.3). Then there exists $m \in \mathbb{N}_{k}$ such that

$$
0<\left|a_{m}\right|<\frac{B-A}{\left|\alpha_{m}\right|} \text { or } 0<\left|b_{m}\right|<\frac{B-A}{\left|\beta_{m}\right|} .
$$

If $0<\left|a_{m}\right|<\frac{B-A}{\left|\alpha_{m}\right|}$, then putting

$$
\gamma=\frac{\left|\alpha_{m} a_{m}\right|}{B-A}, \varphi=\frac{1}{1-\gamma}\left(f-\gamma h_{m}\right),
$$

we have that $0<\gamma<1, h_{m} \neq \varphi$ and

$$
f=\gamma h_{m}+(1-\gamma) \varphi .
$$

Thus, $f \notin E \mathcal{S}_{\mathcal{T}}^{\lambda, \tau}(k, \eta ; A, B)$. Similarly, if $0<\left|b_{m}\right|<\frac{B-A}{\left|\beta_{n}\right|}$, then pouting

$$
\gamma=\frac{\left|\beta_{m} b_{m}\right|}{B-A}, \phi=\frac{1}{1-\gamma}\left(f-\gamma g_{m}\right),
$$

we have that $0<\gamma<1, g_{m} \neq \phi$ and

$$
f=\gamma g_{m}+(1-\gamma) \phi
$$

It follows that $f \notin E \mathcal{S}_{\mathcal{T}}^{\lambda, \tau}(k, \eta ; A, B)$, and the proof is completed. 


\section{Applications}

It is clear that if the class

$$
\mathcal{F}=\left\{f_{n} \in \mathcal{H}: n \in \mathbb{N}\right\},
$$

is locally uniformly bounded, then

$$
\overline{c o} \mathcal{F}=\left\{\sum_{n=1}^{\infty} \gamma_{n} f_{n}: \quad \sum_{n=1}^{\infty} \gamma_{n}=1, \gamma_{n} \geq 0(n \in \mathbb{N})\right\} .
$$

Thus, by Theorem 2.1 we have the following corollary.

\section{Corollary 5.1.}

$$
\mathcal{S}_{\mathcal{T}}^{\lambda, \tau}(k, \eta ; A, B)=\left\{\sum_{n=k-1}^{\infty}\left(\gamma_{n} h_{n}+\delta_{n} g_{n}\right): \sum_{n=k-1}^{\infty}\left(\gamma_{n}+\delta_{n}\right)=1\left(\delta_{k-1}=0, \gamma_{n}, \delta_{n} \geq 0\right)\right\}
$$

where $h_{n}, g_{n}$ are defined by (4.3).

For each fixed value of $m, n \in \mathbb{N}_{k}, z \in \mathbb{U}$, the following real-valued functionals are continuous and convex on $\mathcal{H}$ :

$$
\mathcal{J}(f)=\left|a_{n}\right|, \mathcal{J}(f)=\left|b_{n}\right|, \mathcal{J}(f)=|f(z)| \mathcal{J}(f)=\left|D_{\mathcal{H}} f(z)\right| \quad(f \in \mathcal{H}) .
$$

Moreover, for $\gamma \geq 1,0<r<1$, the real-valued functional

$$
\mathcal{J}(f)=\left(\frac{1}{2 \pi} \int_{0}^{2 \pi}\left|f\left(r e^{i \theta}\right)\right|^{\gamma} d \theta\right)^{1 / \gamma} \quad(f \in \mathcal{H})
$$

is also continuous and convex on $\mathcal{H}$.

Therefore, by Lemma 4.1 and Theorem 2.1 we have the following corollaries.

Corollary 5.2. Let $f \in \mathcal{S}_{\mathcal{T}}^{\lambda, \tau}(k, \eta ; A, B)$ be a function of the form (3.5). Then

$$
\left|a_{n}\right| \leq \frac{B-A}{\left|\alpha_{n}\right|},\left|b_{n}\right| \leq \frac{B-A}{\left|\beta_{n}\right|} \quad\left(n \in \mathbb{N}_{k}\right),
$$

where $\alpha_{n}, \beta_{n}$ are defined by (3.8). The result is sharp. The functions $h_{n}, g_{n}$ of the form (4.3) are the extremal functions.

Corollary 5.3. Let $f \in \mathcal{S}_{\mathcal{T}}^{\lambda, \tau}(k, \eta ; A, B),|z|=r<1$. Then

$$
\begin{aligned}
r-\frac{B-A}{\left|\lambda_{k}\right|(k-1+k B-A)} r^{k} & \leq|f(z)| \leq r+\frac{B-A}{\left|\lambda_{k}\right|(k-1+k B-A)} r^{k}, \\
r-\frac{k(B-A)}{\left|\lambda_{k}\right|(k-1+k B-A)} r^{k} & \leq\left|D_{\mathcal{H}} f(z)\right| \leq r+\frac{k(B-A)}{\left|\lambda_{k}\right|(k-1+k B-A)} r^{k},
\end{aligned}
$$

where $\lambda_{k}$ is defined by (3.5). The result is sharp. The function $h_{k}$ of the form (4.3) is the extremal function.

Corollary 5.4. Let $0<r<1, \gamma \geq 1$. If $f \in \mathcal{S}_{\mathcal{T}}^{\lambda, \tau}(k, \eta ; A, B)$, then

$$
\begin{aligned}
\frac{1}{2 \pi} \int_{0}^{2 \pi}\left|f\left(r e^{i \theta}\right)\right|^{\gamma} d \theta & \leq \frac{1}{2 \pi} \int_{0}^{2 \pi}\left|h_{k}\left(r e^{i \theta}\right)\right|^{\lambda} d \theta, \\
\frac{1}{2 \pi} \int_{0}^{2 \pi}\left|D_{\mathcal{H}} f(z)\right|^{\gamma} d \theta & \leq \frac{1}{2 \pi} \int_{0}^{2 \pi}\left|D_{\mathcal{H}} h_{k}\left(r e^{i \theta}\right)\right|^{\gamma} d \theta,
\end{aligned}
$$

where $h_{k}$ is the function defined by (4.3). 
The following covering result follows from Corollary 5.3.

Corollary 5.5. If $f \in \mathcal{S}_{\mathcal{T}}^{\lambda, \tau}(k, \eta ; A, B)$, then $\mathbb{U}(r) \subset f(\mathbb{U})$, where

$$
r=1-\frac{B-A}{\left|\lambda_{k}\right|(k-1+k B-A)} .
$$

By using Corollary 3.4 and the results above we obtain corollaries listed below.

Corollary 5.6. The class $\mathcal{R}_{\mathcal{T}}^{\lambda, \tau}(k, \eta ; A, B)$ is convex and compact subset of $\mathcal{H}$. Moreover,

$$
E \mathcal{R}_{\mathcal{T}}^{\lambda, \tau}(k, \eta ; A, B)=\left\{h_{n}: n \in \mathbb{N}_{k-1}\right\} \cup\left\{g_{n}: n \in \mathbb{N}_{k}\right\},
$$

and

$$
\mathcal{R}_{\mathcal{T}}^{\lambda, \tau}(k, \eta ; A, B)=\left\{\sum_{n=k-1}^{\infty}\left(\gamma_{n} h_{n}+\delta_{n} g_{n}\right): \sum_{n=k-1}^{\infty}\left(\gamma_{n}+\delta_{n}\right)=1\left(\delta_{k-1}=0, \gamma_{n}, \delta_{n} \geq 0\right)\right\},
$$

where $h_{k-1}(z)=z$, and

$$
h_{n}(z)=z-\frac{(B-A) e^{i(1-n) \eta}}{(1+B) \lambda_{n}} z^{n}, g_{n}(z)=z+\frac{(B-A) e^{i(1+n) \eta}}{(1+B) \tau \overline{\lambda_{n}}} \bar{z}^{n} \quad(z \in \mathbb{U}) .
$$

Corollary 5.7. Let $f \in \mathcal{R}_{\mathcal{T}}^{\lambda, \tau}(k, \eta ; A, B)$ be a function of the form (1.4). Then

$$
\begin{aligned}
& \left|a_{n}\right| \leq \frac{B-A}{(1+B)\left|\lambda_{n}\right|},\left|b_{n}\right| \leq \frac{B-A}{(1+B)\left|\lambda_{n}\right|} \quad\left(n \in \mathbb{N}_{k}\right), \\
& r-\frac{B-A}{(1+B)\left|\lambda_{k}\right|} r^{k} \leq|f(z)| \leq r+\frac{B-A}{(1+B)\left|\lambda_{k}\right|} r^{k} \quad(|z|=r<1), \\
& r-\frac{k(B-A)}{(1+B)\left|\lambda_{k}\right|} r^{k} \leq\left|D_{\mathcal{H}} f(z)\right| \leq r+\frac{k(B-A)}{(1+B)\left|\lambda_{k}\right|} r^{k} \quad(|z|=r<1), \\
& \quad \frac{1}{2 \pi} \int_{0}^{2 \pi}\left|f\left(r e^{i \theta}\right)\right|^{\gamma} d \theta \leq \frac{1}{2 \pi} \int_{0}^{2 \pi}\left|h_{k}\left(r e^{i \theta}\right)\right|^{\lambda} d \theta,
\end{aligned}
$$

where $\lambda_{n}$ is defined by (3.5). The results are sharp. The functions $h_{n}, g_{n}$ of the form (5.5) are the extremal functions.

Corollary 5.8. Let us assume (3.6). If $f \in \mathcal{R}_{\mathcal{T}}^{\lambda, \tau}(k, \eta ; A, B)$, then $\mathbb{U}(r) \subset f(\mathbb{U})$, where

$$
r=1-\frac{B-A}{(1+B)\left|\lambda_{k}\right|} \text {. }
$$

The main classes $\mathcal{S}_{\mathcal{H}}^{\lambda, \tau}(k ; A, B)$ and $\mathcal{R}_{\mathcal{H}}^{\lambda, \tau}(k ; A, B)$ are related to harmonic starlike functions, harmonic convex functions and harmonic Janowski functions.

Let us define

$$
\begin{aligned}
\mathcal{S}_{\mathcal{H}}(k, \alpha): & =\mathcal{S}_{\mathcal{H}}^{0}(k ; 2 \alpha-1,1), \mathcal{K}_{\mathcal{H}}(k, \alpha):=\mathcal{S}_{\mathcal{H}}^{1}(k ; 2 \alpha-1,1), \\
N_{\mathcal{H}}(k, \alpha): & =\mathcal{R}_{\mathcal{H}}^{1}(k ; 2 \alpha-1,1), R_{\mathcal{H}}(k, \alpha):=\mathcal{R}_{\mathcal{H}}^{2}(k ; 2 \alpha-1,1) .
\end{aligned}
$$

The classes $\mathcal{S}_{\mathcal{H}}(\alpha):=\mathcal{S}_{\mathcal{H}}(2, \alpha)$ and $\mathcal{K}_{\mathcal{H}}(\alpha):=\mathcal{K}_{\mathcal{H}}(2, \alpha)$ were investigated by Jahangiri [9] (see also [2,18]). They are the classes of starlike and convex functions of order $\alpha$, respectively. The classes $N_{\mathcal{H}}(\alpha):=N_{\mathcal{H}}(2, \alpha)$ and $N_{\mathcal{H}}(\alpha):=N_{\mathcal{H}}(2, \alpha)$ are studied in [1] (see also [13]). Finally, the classes $\mathcal{S}_{\mathcal{H}}:=\mathcal{S}_{\mathcal{H}}(0)$ and $\mathcal{K}_{\mathcal{H}}:=\mathcal{K}_{\mathcal{H}}(0)$ are the classes of functions which are starlike and convex in $\mathbb{U}(r)$, respectively, for all $r \in(0,1\rangle$. We should notice that the classes $\mathcal{S}(A, B):=\mathcal{S}_{\mathcal{H}}(A, B) \cap \mathcal{A}$ and $\mathcal{R}(A, B):=\mathcal{R}_{\mathcal{H}}(A, B) \cap \mathcal{A}$ were introduced by Janowski [12].

Using Theorem 2.3 or Theorem 2.4 for the classes defined above we obtain corollaries listed below. 


\section{Corollary 5.9.}

$$
\mathcal{S}_{\mathcal{H}}(k, \alpha)=\left\{\psi_{\xi}:|\xi|=1\right\}^{*}
$$

where

$$
\psi_{\xi}(z):=z \frac{2(1-\alpha) \xi+(1-\xi+2 \alpha \xi) z}{(1-z)^{2}}-\bar{z} \frac{2+2 \alpha \xi-(1-\xi+2 \alpha \xi) \bar{z}}{(1-\bar{z})^{2}} \quad(z \in \mathbb{U}) .
$$

Corollary 5.10.

$$
\mathcal{K}_{\mathcal{H}}(k, \alpha)=\left\{\psi_{\xi}:|\xi|=1\right\}^{*}
$$

where

$$
\psi_{\xi}(z):=2 z \frac{(1-\alpha) \xi+(1+\alpha \xi) z}{(1-z)^{3}}+2 \bar{z} \frac{1+\alpha \xi+(1-\alpha) \xi \bar{z}}{(1-\bar{z})^{3}} \quad(z \in \mathbb{U}) .
$$

Corollary 5.11.

$$
N_{\mathcal{H}}(k, \alpha)=\left\{\delta_{\xi}:|\xi|=1\right\}^{*},
$$

where

$$
\delta_{\xi}(z):=z \frac{2(1-\alpha) \xi+(2 \alpha \xi-\xi+1)\left(z^{2}-2 z\right)}{(1-z)^{2}}-\bar{z} \frac{1+\xi}{(1-\bar{z})^{2}} \quad(z \in \mathbb{U}) .
$$

Corollary 5.12. [4]

$$
\mathcal{S}_{\mathcal{H}}^{*}=\left\{\psi_{\xi}:|\xi|=1\right\}^{*},
$$

where

$$
\psi_{\xi}(z):=z \frac{2 \xi+(1-\xi) z}{(1-z)^{2}}-\bar{z} \frac{2-(1-\xi) \bar{z}}{(1-\bar{z})^{2}} \quad(z \in \mathbb{U}) .
$$

Corollary 5.13. [4]

$$
\mathcal{K}_{\mathcal{H}}=\left\{\psi_{\xi}:|\xi|=1\right\}^{*}
$$

where

$$
\psi_{\xi}(z):=2 z \frac{\xi+z}{(1-z)^{3}}+2 \bar{z} \frac{1+\xi \bar{z}}{(1-\bar{z})^{3}} \quad(z \in \mathbb{U}) .
$$

The class $\mathcal{S}_{\mathcal{T}}^{\lambda, \tau}(k, \eta ; A, B)$ generalizes the classes of starlike functions of complex order. Let us define

$$
\mathcal{e} \mathcal{S}_{\mathcal{H}}(k, \gamma):=\mathcal{S}_{\mathcal{H}}(k ; 1-2 \gamma, 1) \quad(\gamma \in \mathbb{C} \backslash\{0\}) .
$$

The class $\mathcal{C S}_{\mathcal{H}}(\gamma):=\mathcal{C} \mathcal{S}_{\mathcal{H}}(2, \gamma)$ was defined by Yalçın and Öztürk [23]. In particular, if we put $\gamma:=\frac{1-\alpha}{1+e^{i \eta}}$, then we obtain the class $\mathcal{R} \mathcal{S}_{\mathcal{H}}(\alpha, \eta):=\mathcal{S}_{\mathcal{H}}\left(\frac{2 \alpha-1+e^{i \eta}}{1+e^{i \eta}}, 1\right)$ studied by Yalçin et al. [24]. It is the class of functions $f \in \mathcal{S}_{\mathcal{H}}(k)$ such that

$$
\operatorname{Re}\left\{\left(1+e^{i \eta}\right) \frac{D_{\mathcal{H}} f(z)}{f(z)}-e^{i \eta}\right\}>\alpha \quad(z \in \mathbb{U}, \eta \in \mathbb{R}) .
$$

By Theorem 2.3 we have the following corollary.

\section{Corollary 5.14.}

$$
\mathcal{C S}_{\mathcal{H}}(k, \gamma)=\left\{\psi_{\xi}:|\xi|=1\right\}^{*}
$$

where

$$
\psi_{\xi}(z):=z \frac{2 \gamma \xi+(1+\xi-2 \gamma \xi) z}{(1-z)^{2}}-\bar{z} \frac{2+2(1-\gamma) \xi-(1+\xi-2 \gamma \xi) \bar{z}}{(1-\bar{z})^{2}} \quad(z \in \mathbb{U}) .
$$

Corollary 5.15. [5]

$$
\operatorname{CS}_{\mathcal{H}}^{n}(\alpha, \eta)=\left\{\psi_{\xi}:|\xi|=1\right\}^{*}
$$

where $\psi_{\xi}$ is defined by (5.6) with $\gamma:=\frac{1-\alpha}{1+e^{i \eta}}$. 
Remark 5.16. By varying the parameters in the defined classes of functions we can obtain new and also well-known results (see for example [1-7, 9-13, 18, 21-24]).

Acknowledgment. The work is supported by the Centre for Innovation and Transfer of Natural Sciences and Engineering Knowledge, University of Rzeszów.

\section{References}

[1] O. Ahuja, Connections between various subclasses of planar harmonic mappings involving hypergeometric functions, Appl. Math. Comput. 198, 305-316, 2008.

[2] O.P. Ahuja and J.M. Jahangiri, Certain multipliers of univalent harmonic functions, Appl. Math. Lett. 18, 1319-1324, 2005.

[3] M. Darus and K. Al-Shaqsi, K. On certain subclass of harmonic univalent functions, J. Anal. Appl. 6, 17-28, 2008.

[4] J. Dziok, On Janowski harmonic functions, J. Appl. Anal. 21, 99-107, 2015.

[5] J. Dziok, Ruscheweyh-type harmonic functions with correlated coefficients, Filomat, 33 (12), 2019.

[6] J. Dziok, J.M. Jahangiri, and H. Silverman, Harmonic functions with varying coefficients, J. Inequal. Appl. 139, 2016.

[7] J. Dziok, M. Darus, J. Sokół, and T. Bulboaca, Generalizations of starlike harmonic functions, C. R. Math. Acad. Sci. Paris, 354, 13-18, 2016.

[8] D.J. Hallenbeck and T.H. MacGregor, Linear Problems and Convexity Techniques in Geometric Function Theory, Pitman Advanced Publishing Program, Boston, Pitman, 1984.

[9] J.M. Jahangiri, Harmonic functions starlike in the unit disk, J. Math. Anal. Appl. 235, 470-477, 1999.

[10] J.M. Jahangiri, G. Murugusundaramoorthy, and K. Vijaya, Starlikeness of harmonic functions defined by Ruscheweyh derivatives, J. Indian Acad. Math. 26, 191-200, 2004.

[11] J.M. Jahangiri and H. Silverman, Harmonic univalent functions with varying arguments, Int. J. Appl. Math. 8, 267-275, 2002.

[12] W. Janowski, Some extremal problems for certain families of analytic functions I, Ann. Polon. Math. 28, 297-326, 1973.

[13] S.Y. Karpuzogullari, M. Öztürk, and M. Yamankaradeniz, A subclass of harmonic univalent functions with negative coefficients, Appl. Math. Comput. 142 (2-3), 469476, 2003.

[14] M. Krein and D. Milman, On the extreme points of regularly convex sets, Studia Mathematica 9, 133-138, 1940.

[15] H. Lewy, On the non-vanishing of the Jacobian in certain one-to-one mappings, Bull. Amer. Math. Soc. 42, 689-692, 1936.

[16] P. Montel, Sur les families de functions analytiques qui admettent des valeurs exceptionelles dans un domaine, Ann. Sci. Ecole Norm. Sup. 23, 487-535, 1912.

[17] G. Murugusundaramoorthy and K. Vijaya, On certain classes of harmonic functions involving Ruscheweyh derivatives, Bull. Calcutta Math. Soc. 96, 99-108, 2004.

[18] M. Öztürk, S. Yalçin, and M. Yamankaradeniz, Convex subclass of harmonic starlike functions, Appl. Math. Comput. 154, 449-459, 2004.

[19] S. Ruscheweyh, Convolutions in geometric function theory, in: Sem. Math. Sup. 83, Les Presses del'Université de Montréal, 1982.

[20] S. Ruscheweyh, New criteria for univalent functions, Proc. Amer. Math Soc. 49, 109-115, 1975.

[21] H. Silverman, Harmonic univalent functions with negative coefficients, J. Math. Anal. Appl. 220, 283-289, 1998. 
[22] S. Yalçın, S.B. Joshi, and E. Yaşar, On certain subclass of harmonic univalent functions defined by a generalized Ruscheweyh derivatives operator, Appl. Math. Sci. (Ruse) 4, 327-336, 2010.

[23] S. Yalçın and M. Öztürk, Harmonic functions starlike of the complex order, Mat. Vesnik 58, 7-11, 2006.

[24] S. Yalçın, M. Öztürk, and M. Yamankaradeniz, On the subclass of Salagean-type harmonic univalent functions, J. Inequal. Pure Appl. Math. 8, Art. 54, 9 pp., 2007. 